\title{
Stress resistance to emotional tension condition in elite athletes
}

\begin{abstract}
The characteristics of stress resistance and emotional tension in elite athletes were studied. 19 elite athletes (12 athletes at age of 19-24 and 7 athletes aged 27-31); members of the Ukrainian National Team in Greco-Roman wrestling took part in the research. The balance of nervous system, stress tolerance and state of autonomic regulation of the heart rhythm were studied. The results of the study are shows that athletes in younger age group (1924) have higher level of performance of cognitive functions. Apart of it, it was indicated that athletes in a younger age group has the balance of nervous processes to acceleration and deceleration. Psycho emotional load leads to decline of average meanings of cardio intervals (Mean RR), the increase of low-frequency (VLF) and high-frequency (HF) heart rate variations in older age group. This fact indicates the influence to sinus node the central contour of regulation in the situations of psycho emotional tension with simultaneous activation parasympathetic link of vegetative regulation.
\end{abstract}

Keywords: stress, resistance, elite athletes, emotional tension
Volume I Issue | - 2014

\author{
Korobeynikov G, Korobeinikova L \\ National University of Physical Education and Sport, Ukraine
}

Correspondence: Georgiy Korobeynikov, National University of Physical Education and Sport, Kiev, Ukraine,

Email george.65@mail.ru

Received: April 12, 2014 | Published: April 26, 2014

\section{Introduction}

One of the ways of the study of sport medicine is investigation of resistance of organism of athletes to extremely situations in sport activity. Sports activities as extreme kind of human activities are connected with the presence of psycho emotional factors which can influence the efficiency of sport results. ${ }^{1,2}$ Considering that psychophysiological functions constitute a major link of formation of psycho-emotional reactions in the situations of extreme conditions, it is logical to expect the connection between the athlete's age and the level of stress resistance, indicators of perception and processing of visual information. However, sports activities, as an extreme variety of human activities related to the presence of psycho-emotional factors that influence the effectiveness of athletic performance. ${ }^{3,4}$ In some works it is indicated that stress resistance to renders influences the capacity to tolerate training loads and ability of training of elite athletes. ${ }^{5-7}$ The reason of the stress is a multifactor syndrome which influences to reduction of productivity of athletes. ${ }^{8}$ Hartmann $\&$ Mester $^{9}$ state that the one of the major links of the decreasing capability is imbalance between stress and recovery after loads. With the increasing of qualification of athletes the speed of visual response is increased it the order of magnitude. At the same time, the manifestation of neural and psychomotor abilities of athletes in the competition depends on the functional state of the organisms. ${ }^{10,11}$ In last decade Olympic sports are characterized by the presence of older athletes who have reached high athletic results. The age of champions and runners-up of International championships in some Olympic sports could be 36-42 years in individual sports and up to 52 in such sports as horseback riding and sailing. But some investigations indicate that age-related muscle atrophy that begins at about 50 years of age for elite athletes in all sports. ${ }^{12}$ Despite the large number of studies in the field of stressors in the sport, there are no results about stress resistance of elite athletes of different ages.

The purpose: Study the stress resistance to emotional tension condition in elite athletes.

\section{Methods}

19 elite athletes, members of the Ukrainian National Team in Greco-Roman wrestling took part in the research. The athletes who participated in the study have high level of sport qualification. All of the athletes are the winners of Ukrainian National Championship and seven athletes are the winners of European and World Championships. The athletes were divided in two groups according to their age. 12 athletes at age of 19-24 were placed in one group and 7 athletes aged 27-31 in another group. All of the athletes participated in this study after giving their informed consent. The experimental study was approved by the Ethics Committees for Biomedical Research with accordance the ethical standards of the Helsinki Declaration. Each subject completed all test blocks in one day. To determine the balance between acceleration and deceleration of the central nervous system (CNS) we used the methodology called "Reaction to the Moving Object". In this test all examined athletes were offered 2 pointersdynamic (target) and static (marker), and each athlete could react by sending a discreet and timely signal to the monitor. The level of psycho-emotional resistance (stress tolerance) was determined by the results of test called "Stress Test" with analysis of information regarding the positioned selection of objects in appropriate cells in adopted mode. Thus, the certain time limit for selection of objects is reached and this creates psycho-emotional informational tension (load). The results of the tests allowed determining the criteria of stress resistance, capacity and impulsiveness. All of the methodologies are included in apparatus-program psycho-diagnostic complex "Multipsychometr-05". The estimation of autonomic regulation of the heart rhythm was performed using cardiomonitor «Polar-S800» with the registration of the spectral characteristics of heart rate. The analysis of non-stationary transient system of regulation of heart rate analysis the scattergrams as a non-parametric method of analysis was used. ${ }^{13}$ Determine the parameters SD1 (display aperiodic fluctuations of heart rate) and SD2 (slow oscillations of the heart rhythm).

The experimental study was approved by the Ethics Committees for Biomedical Research with accordance the ethical standards of 
the Helsinki Declaration. Consents for research in writing form were given by the athletes according to the recommendations to Ethics Committees for Biomedical Research. Statistical analysis was performed with the help of programming package Statgraphics 5.1 (Manugistics, Inc.). Since the data obtained in research didn't correspond to the normal distribution of studied data, the methods of nonparametric statistics of Wilcoxon rank-sum test were applied. To reflect data distribution we used interquartile range, thus specifying first quarter $(25 \%)$ and third quarter $(75 \%)$.

\section{Results}

The Table 1 illustrates the data according to methodology "Nervous Process Balance" demonstrated by athletes of different age groups. The data of Table-1 analysis indicates that there are no actual distinction between age groups in the measurements of accuracy and stability. It means that age component doesn't really matter in the efficiency of execution of the tasks with external stimulus in conditions of psycho-emotional stress. According to actual scale, median of acceleration in the first age group reflects the balance of acceleration and deceleration of nervous processes. In the second age group, acceleration parameter indicates the prevalence of acceleration of nervous processes. Therefore, athletes in a younger age group (19-24years) have the balance of nervous processes of acceleration and deceleration. This balance is in agreement with the presence of higher productivity of visual perception and visual information processing efficiency comparing with older age group (27-31years). Consequently, prevalence of acceleration processes in older age group leads to deterioration of visual information perception and processing. The conducted analysis of correlation between age and data which reflecting the balance of nervous processes in different age groups showed higher coefficient values of correlation in older age group. Hence, the correlation coefficient between age and stability in younger age group was $r=0,52, p<0,05$, while in older age group this criterion was $r=0,87, p<0,05$. Likewise, correlation coefficient between age and acceleration in younger age group was $r=0,43, p<0,05$, while in older age group it was higher and amounted to $r=0,65, p<0,05$.

Table I Medians of variables of balance of nervous processes in different age groups $(n=19)$

\begin{tabular}{|c|c|c|c|c|c|c|}
\hline \multirow[t]{2}{*}{ Variables } & \multicolumn{3}{|c|}{ First age group $(n=\mid 2)$} & \multicolumn{3}{|c|}{ Second age group $(n=7)$} \\
\hline & Median & Lower quarter & Upper quarter & Median & Lower quarter & Upper quarter \\
\hline Accuracy (secret unit) & 2,76 & $2,4 I$ & 3,04 & 3,97 & 2,86 & 4,85 \\
\hline Stability, cV (\%) & 3,28 & 3,02 & 3,96 & 3,00 & 2,55 & 4,57 \\
\hline Acceleration (secret unit) & $-0,28$ & $-1,10$ & 0,37 & $-1,27^{*}$ & $-3,60$ & 0,01 \\
\hline
\end{tabular}

Note: ${ }_{-}-p<0,05$, comparing with the first age group of the athletes.

We believe that deterioration in the state of neurodynamic functions in the situations of psycho-emotional stress in the older age group of elite athletes is not so much the deterioration of afferent compound of perception system, information analysis and processing, but in fact efferent motor compound. Besides, with aging the connection between effectiveness of visual perception and information processing are improves. ${ }^{13}$

The Table 2 reflects the data of stress resistance in different age groups. According to the Table-2, there is no actual distinction in variables of general intensity and impulsiveness in different age groups. At the same time, there is actual distinction in variables of stress resistance in different age groups. Stress resistance is determined by the ratio of average capacity of visual analyzer at the beginning of the test to the capacity in the end of the test. In other words, the variables of stress resistance indicate the possibility of maintaining

Table 2 Medians of variables of stress resistance in different age groups $(n=19)$

\begin{tabular}{|c|c|c|c|c|c|c|}
\hline \multirow[t]{2}{*}{ Variables } & \multicolumn{3}{|c|}{ First age group $(n=\mid 2)$} & \multicolumn{3}{|c|}{ Second age group $(n=7)$} \\
\hline & Median & Lower quarter & $\begin{array}{l}\text { Upper } \\
\text { quarter }\end{array}$ & Median & Lower quarter & $\begin{array}{l}\text { Upper } \\
\text { quarter }\end{array}$ \\
\hline Stress Resistance (secret unit) & 88,27 & 79,01 & 90,33 & $109,20 *$ & 102,83 & 118,35 \\
\hline General Efficiency (secret unit) & 1,09 & $\mathrm{I}, 07$ & 1,13 & 1,10 & 0,92 & I, I5 \\
\hline Impulsiveness (secret unit) & $-0,04$ & $-0,06$ & 0,00 & $-0,03$ & $-0,06$ & 0,00 \\
\hline
\end{tabular}

Note: $*_{-p}<0,05$, comparing with the first age group of the athletes.

The Table 3 reflects the medians of spectral characteristics of heart rate variability in the beginning and in the end of psycho emotional tension in different age groups. Analysis of data of Table 3 confirms that there is an actual distinction in heart rate variability HF and of the sufficient level of capacity of visual sensor system in situations of psycho emotional stress. Thus, the athletes of a younger age group showed better results of stress resistance comparing with older age group. The conducted analysis of correlation between athletes' age and stress resistance indicates the presence of actual correlation coefficients only in variables of stress $(r=0,42, p<0,05)$ in younger age group. The established fact confirms the connection between age and data of stress resistance to psycho-emotional stress. The athletes of older age group didn't show actual connection between age and stress resistance. Therefore, the conducted research of elite athletes confirmed that there is age deterioration in stress resistance capacity in situations of psycho-emotional stress. Spectral characteristics of cardio intervals were studied to determine the age distinctiveness of heart rate vegetation regulation in situations of psycho-emotional stress.
LF/HF between different age groups in the beginning of psychoemotional loading. Actually higher variables of HF confirm the preeminence of parasympathetic activation of heart rate regulation in younger age group. The reduced of variables of LF/HF in younger 
age group indicate the optimization of vegetative balance between sympathetic and parasympathetic tone, Psycho- emotional loading leads to decreasing of average meanings of cardio intervals (Mean
RR), the increase of low- frequency (VLF) and high-frequency (HF) heart rate variations in older age group.

Table 3 Medians of spectral characteristics of heart rate variability showed by athletes of different age groups in dynamic of psycho emotional $(n=19)$

\begin{tabular}{|c|c|c|c|c|c|c|c|}
\hline \multirow[t]{2}{*}{ Variables } & & \multicolumn{3}{|c|}{ First age group $(n=\mid 2)$} & \multicolumn{3}{|c|}{ Second age group $(n=7)$} \\
\hline & & Median & $\begin{array}{l}\text { Lower } \\
\text { quarter }\end{array}$ & $\begin{array}{l}\text { Upper } \\
\text { quarter }\end{array}$ & Median & $\begin{array}{l}\text { Lower } \\
\text { quarter }\end{array}$ & $\begin{array}{l}\text { Upper } \\
\text { quarter }\end{array}$ \\
\hline \multirow[t]{2}{*}{ Mean RR (ms) } & in the beginning & 1034,25 & 455,18 & 1202,70 & 1009,50 & 1008,70 & 1156,60 \\
\hline & in the end & 901,15 & 469,90 & 995,25 & $78 I, 40 \&$ & 781,40 & $87 I, 70$ \\
\hline \multirow[t]{2}{*}{ VLF (ms2) } & in the beginning & 4285,00 & 1396,50 & 10839,50 & 9239,00 & 4802,00 & 10398,00 \\
\hline & in the end & 3262,00 & 2598,50 & 8553,50 & $1722,00 \&$ & 1722,00 & 1977,00 \\
\hline \multirow[t]{2}{*}{ LF (ms2) } & in the beginning & 2405,00 & 1785,50 & 2591,00 & 2474,00 & 2428,00 & 3906,00 \\
\hline & in the end & 1924,00 & 1558,50 & 3359,50 & 2843,00 & 1400,00 & 2843,00 \\
\hline \multirow[t]{2}{*}{$\mathrm{HF}(\mathrm{ms} 2)$} & in the beginning & 2166,00 & 1358,00 & 2697,00 & $1428,00 *$ & 1276,00 & 2586,00 \\
\hline & in the end & 1199,50 & 517,00 & 2808,00 & $2843,00 * \&$ & 1400,00 & 2843,00 \\
\hline \multirow[t]{2}{*}{ Total } & in the beginning & 11856,00 & 4483,00 & 19317,00 & 14103,00 & 11294,00 & 14853,00 \\
\hline & in the end & 5257,00 & 4430,00 & 20228,00 & $4887,00 \&$ & 3849,00 & 4887,00 \\
\hline \multirow[t]{2}{*}{ LF/HF } & in the beginning & $\mid, 21$ & 0,70 & 2,30 & $\mathrm{I}, 73^{*}$ & I, $5 \mathrm{I}$ & 1,90 \\
\hline & in the end & $2,382 \&$ & I, 296 & 3,96 & $8,8|| * \&$ & 2,966 & 8,81 \\
\hline
\end{tabular}

Notes: *I.*-p $<0,05$, comparing with the first age group of the athletes.

2. \& $p<0,05$, comparing to the beginning of the loading.

This fact indicates the influence to central contour of regulation of sinus node in the situations of psycho- emotional tension with simultaneous activation parasympathetic link of vegetative regulation and renin-angiotensin-aldosterone system. This fact correlated with the shift of vegetative balance (LF/HF) towards sympathetic activation of vegetative regulation of cardio intervals. Thus, psycho emotional tension of athletes in older age group causes significant changes in heart rate variability. In younger age group were the registered the changes of vegetative balance $(\mathrm{LF} / \mathrm{HF})$, that indicates the amplifications of sympathetic activation of heart rate regulation, for comparison with the older age group. This indicates the optimal reaction of autonomic regulation system to psycho-emotional tension.

\section{Discussion}

The study shows that athletes in younger age group (19-24years) has more productive visual perception and higher efficiency of visual information processing comparing with older age group (27-31years). It indicates the connection between elite athletes' age and activity of cognitive functions. Apart of it, it was indicated that athletes in a younger age group has the balance of nervous processes to acceleration and deceleration. It correlated with the presence of higher productivity of visual perception and visual information processing efficiency comparing with older age group (27-31years).

The results correlated with our previous study the deterioration in the state of neurodynamic functions in the situations of psychoemotional stress in the older age group of elite athletes, this process of deterioration of efferent part of information processing of elite athletes during aging process. Analogical results show that, the athletes of a younger age group showed better results of stress resistance comparing with older age group. The study of heart rate variability shows that psycho-emotional tension of athletes in older age group causes significant changes in heart rate variability signifying the stress type of loading. In younger age group the changes of vegetative balance were indicated as optimal reaction of vegetative regulation system to psycho-emotional tension.

\section{Acknowledgments}

None.

\section{Conflicts of interest}

Authors declare that there is no conflict of interest.

\section{References}

1. Feltz DL, Lirgg CD. Perceived team and player efficacy in hockey. $J$ Appl Psychol. 1998;83(4):557-564.

2. Tauer JM, Harackiewicz JM. The Effects of Cooperation and Competition on Intrinsic Motivation and Performance. J Pers Soc Psychol. 2004;86(6):849-861.

3. Robin SV. Mental Skills Training in Sport. In: Gershon T \& Robert CE (Eds.), Handbook of Sport Psychology. 3rd edition. New York, USA: John Wiley \& Sons; 2007. p. 287-309.

4. Korobeynikov G, Mazmanian K, Korobeynikova L, et al. Psycho physiological states and motivation in elite judokas. Arch Budo. 2010;6(3):129-136.

5. Eduardo D, Gonzalez L. Successful technical-tactical combinations in the standing position. International Journal of Wrestling Science. 2011;1(2):19-25. 
6. Brent SR. A tool for measuring stress tolerance in elite athletes. Journal of Applied Sport Psychology. 1990;2(1):51-62.

7. Freitas CG, Aoki MS, Schultz Arruda AF, et al. Training load, stress tolerance and upper respiratory tract infection in basketball players. Brazilian Jourmal of Kinanthropomerty and Human Performance. 2013;15(1): 49-59.

8. Kraemer W, Duncan ND, Volek JS. Resistance Training and Elite Athletes: Adaptations and program Considerations. J Orthop Sports Phys Ther. 1988;28(2):110-119.

9. Hartmann U, Mester J. Training and overtraining markers in selected sports events. Med Sci Sports Exer. 2000;32(1):209-215.
10. Abernethy B, Neal RJ. Visual characteristics of clay target shooters. J Sci Med Sport. 1999;2(1):1-19.

11. Guttmann MC, Pollock M, Foster R, Schmidt D. Training stress in Olympic speed skaters: a psychological perspective. Physician Sports Medicine. 1984;12:45-57.

12. Faulkner JA, Davis CS, Mendias CL, et al. The aging of elite male athletes: age-related changes in performance and skeletal muscle structure and function. Clin J Sport Med. 2008;18(6):501-507.

13. Korobeynikov G, Korobeynikova L, Chernozub A, Makarchuk M. The autonomic regulation of heart rate of athletes with different levels of sensor motor response. J Clin Exp Cardiolog. 2013;4:262. 